\title{
Anisotropic spin-wave patterns generated by spin-torque nano-oscillators
}

Cite as: J. Appl. Phys. 109, 07 C733 (2011); https://doi.org/10.1063/1.3566000

Submitted: 24 September 2010 . Accepted: 11 January 2011 . Published Online: 11 April 2011

F. Macià, A. D. Kent, and F. C. Hoppensteadt

ARTICLES YOU MAY BE INTERESTED IN

The design and verification of MuMax3

AIP Advances 4, 107133 (2014); https://doi.org/10.1063/1.4899186

Multi-frequency magnonic logic circuits for parallel data processing

Journal of Applied Physics 111, 054307 (2012); https://doi.org/10.1063/1.3689011

Conceptual design of spin wave logic gates based on a Mach-Zehnder-type spin wave interferometer for universal logic functions

Journal of Applied Physics 104, 053909 (2008); https://doi.org/10.1063/1.2975235

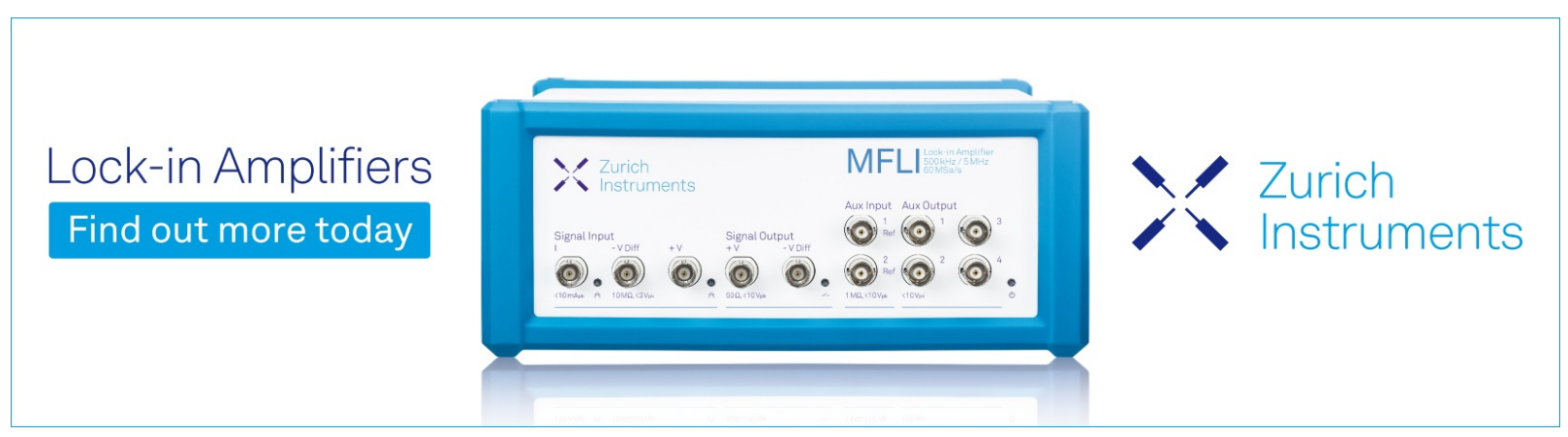




\title{
Anisotropic spin-wave patterns generated by spin-torque nano-oscillators
}

\author{
F. Macià, ${ }^{1,2, a)}$ A. D. Kent, ${ }^{1}$ and F. C. Hoppensteadt ${ }^{2}$ \\ ${ }^{1}$ Department of Physics, New York University, 4 Washington Place, New York, New York 10003, USA \\ ${ }^{2}$ Courant Institute of Mathematical Sciences, New York University, 251 Mercer Street, New York, \\ New York 10012, USA
}

(Presented 17 November 2010; received 24 September 2010; accepted 11 January 2011; published online 11 April 2011)

\begin{abstract}
Spin-wave excitations due to spin-momentum transfer in ferromagnetic thin films will enable new types of information processing and memory storage. Here, we show how arrays of spin-torque nanooscillators (STNOs) can be used to create anisotropic spin-wave interference patterns, which can be used for information processing. We consider STNO arrays contacting a thin ferromagnetic film. Contacts to the film (including the STNOs themselves) can be used to detect the spin-waves and then, when coupled to a simple circuit, can create new excitation patterns. The propagating spin-wave patterns can be generated by pulsing transponders. Arrangements of transponders create resonant (reverberating) spin-wave activity - that may be the basis of polychronous wave computation of the arithmetic and Boolean functions as well as information storage. (C) 2011 American Institute of Physics. [doi:10.1063/1.3566000]
\end{abstract}

Spin-torque nano-oscillators (STNOs) consist of a point contact to a magnetic thin film multilayer, ${ }^{1-6}$ a free and fixed magnetic layer separated by a non-magnetic layer. In such structures, due to the spin-transfer effect, ${ }^{4-6} \mathrm{dc}$ current densities greater than a critical value $\left(j>j_{c}\right)$ generate a high-frequency dynamic response $(1-100 \mathrm{GHz})$ in the free ferromagnetic (FM) layer and result in the emission of spinwaves. These spin-wave excitations have been inferred from the $I-V$ characteristics of the contacts as well as their noise spectra. ${ }^{6}$ Phase locking between proximal STNOs oscillators has also been observed for distances up to $500 \mathrm{~nm},{ }^{7-9}$ and the main coupling mechanism responsible for phase locking is thought to be through spin-waves that propagate between the contacts. ${ }^{10}$

Of interest is control of the direction of spin-wave propagation for a variety of purposes, including on-chip communication or information processing using waves. ${ }^{11}$ STNOs are interesting for such applications because they are highly localized spin-wave sources, in contrast to spin-waves generated using microwave antennas with rf magnetic fields. In this paper we show that multiple frequency locked STNO contacts result in anisotropic interference patterns, enhancing spin-waves activity in some regions and damping activity in others. We further illustrate how such patterns can be used to implement polychronous wave computation. ${ }^{12}$

The response to a (polarized) current in a free magnetic layer is phenomenologically described with an additional current-dependent positive damping term in the LandauLifshitz-Gilbert equation. ${ }^{4,5}$ The magnetic excitations have a frequency that depends on the local field in the contact and depends nonlinearly on the amplitude of the excitation. ${ }^{13}$

The oscillation of the magnetic moments is in the plane perpendicular to the effective magnetic field [i.e., the magnitude of the magnetic moment along the effective magnetic field direction is time independent for dc currents, see

\footnotetext{
${ }^{\text {a)} E l e c t r o n i c ~ m a i l: ~ f e r r a n . m a c i a @ g m a i l . c o m . ~}$
}

Fig. 1(b)]. If we consider a unit vector to represent the direction of the magnetization, $\tilde{m}$, then in the geometry considered in Fig. 1 (with the field perpendicular to the $x y$ plane), the out-of-plane magnetic moment, $m_{z}$, does not oscillate and it represents the envelope of the modulated in-plane components, $m_{x}$ and $m_{y},\left(m_{z}^{2}=1-\left(m_{x}^{2}+m_{y}^{2}\right)=1-m^{2}\right)$, we call $m=\left(m_{x}, m_{y}\right)$ the in-plane component, for convenience). Note that the in-plane components have an oscillating nature in addition to the diffusive pattern $\left(m(\rho, t)=e^{i \omega t} \phi(\rho)\right)$; the out-of-plane component does not. Figure 1 depicts the magnetization for a small amplitude excitations, $a \ll 1$ is the maximum amplitude form; in (c) the oscillating in-plane component $m_{x}$ and in (d) the amplitude of the excitation, $|m|$. Figures $1(\mathrm{c})$ and $1(\mathrm{~d})$ correspond to the solution of the linearized LLG equation with spin torque for a dc current in the nanocontact, ${ }^{6}$ which can be expressed in terms of Bessel functions.

The patterns shown in Figs. 1 and 2 are computed for a dc current. Applying a current pulse to an STNO creates diffusing spin-wave excitations in the magnetic layer. A single circular STNO produces a radially symmetric excitation that spreads and damps out as it moves away from the contact (see Fig. 3). Multiple point-contacts create multiple spinwave packets (see Fig. 4).

Multiple contacts subject to current pulses (i.e., arrangements of STNOs spiking at the same time) ignite multiple spin-waves that interfere and enhance activity in preferred directions in the film's plane. In this case, contact geometry, size, and distance can be used to control spin-wave patterns created by arrays of STNOs. Micromagnetic modeling of spin-waves excited by rf-magnetic fields has been done on waveguides ${ }^{14}$ and on double STNOs with boundary conditions. ${ }^{15-17}$ Figure 4 shows spatial-dependent excitations of magnetic moments (amplitude $|m|$ is plotted), in a ferromagnetic thin film in response of an initial pulse, at time $\tau_{0}$, applied to a three STNOs in a triangular arrangement. In Fig. 4 the three point-contacts were simultaneously excited with a current pulse producing a magnetic excitation that diffuses and 
(a)

(c)
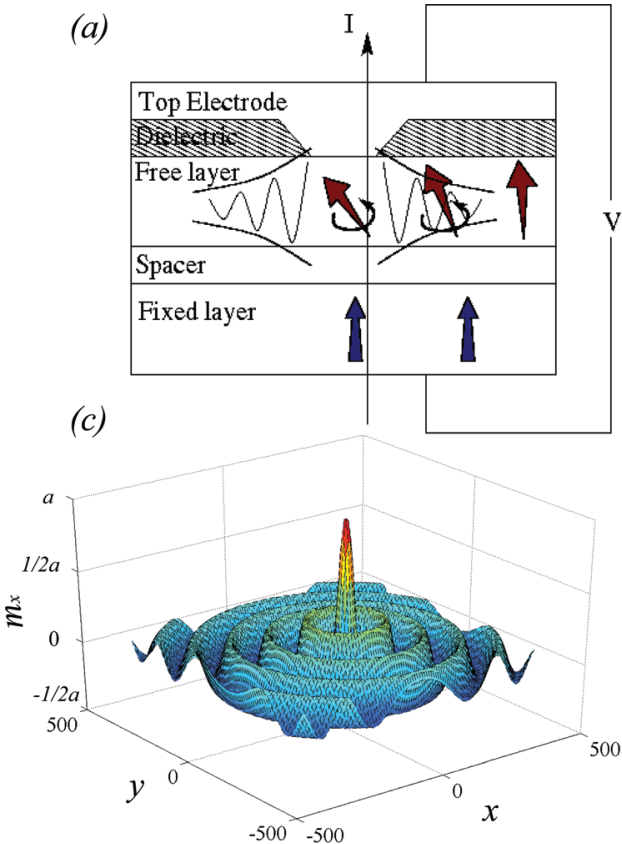

(b)

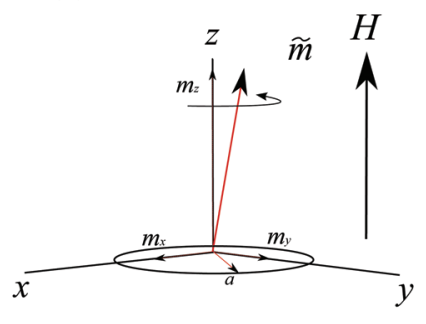

(d)

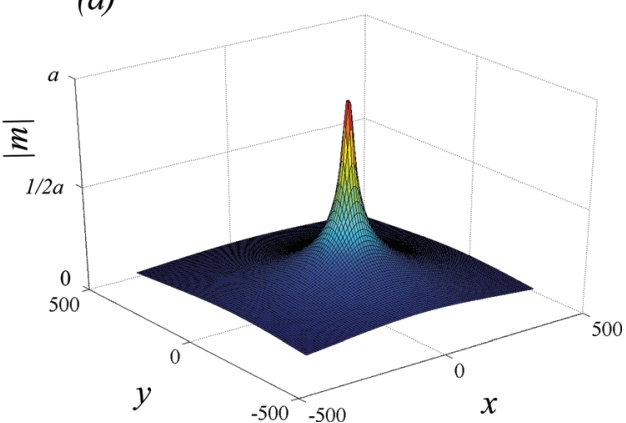

FIG. 1. (Color online) (a) Schematic of an STNO. The charge flows through a point contact to a thin ferromagnetic layer. The magnetic moments in the contact area precess, exciting spin-waves that diffuse in the film. Spatial dependence of the in-plane component, $m_{x}$, is plotted in (c) as well as the amplitude of the excitation, $|m|^{2}=m_{x}^{2}+m_{y}^{2}$, in (d). Results are based on a 800 by $800 \mathrm{~nm}$ film with point-contacts of $20 \mathrm{~nm}$ diameter with dc current. interferes throughout the two-dimensional thin film. Spatialdependent amplitudes, $|m|$, are plotted at times $\tau_{1}, \tau_{2}>\tau_{0}$.

The ability to create propagation spin-wave interference patterns enables a new type of information processing known as polychronous wavefront computation (PWC). ${ }^{11}$ The PWC idea is to encode information into time delays of propagating waves and accomplishes Boolean operations using constructive interference of wave packets. The key ingredients for PWC are a medium that can support interference patterns of propagating activity packets and transponders that can sense the size of incident activity and respond to super-threshold inputs by generating a propagating wave. The propagation times of peak-wave interference correspond to conductiontime delays. The analogy to excitation of a neuron is excitation of a transponder that may fire when a certain threshold

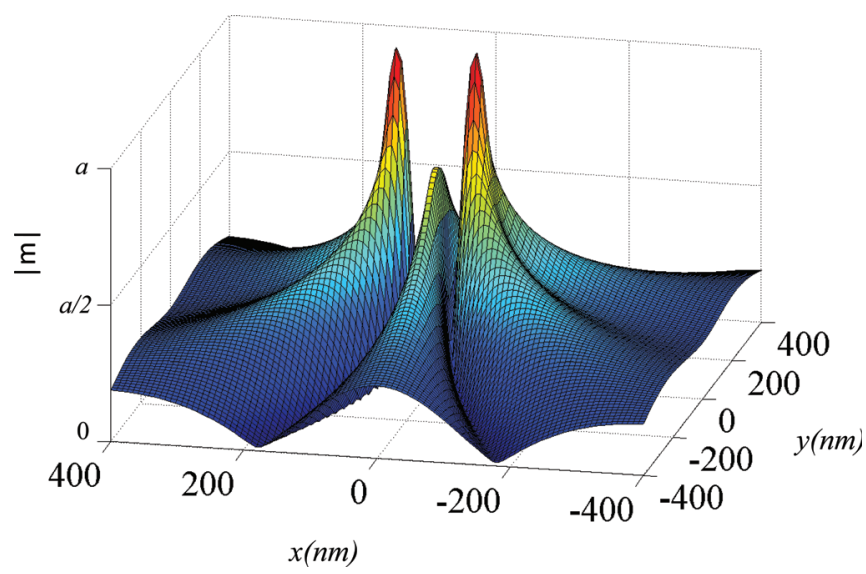

FIG. 2. (Color online) Spatial dependence of the amplitude of the excitation, $|m|^{2}=m_{x}{ }^{2}+m_{y}{ }^{2}$. Results are based on a 800 by $800 \mathrm{~nm}$ film with pointcontacts of $20 \mathrm{~nm}$ diameter, and separation between contacts of $60 \mathrm{~nm}$ with dc current.
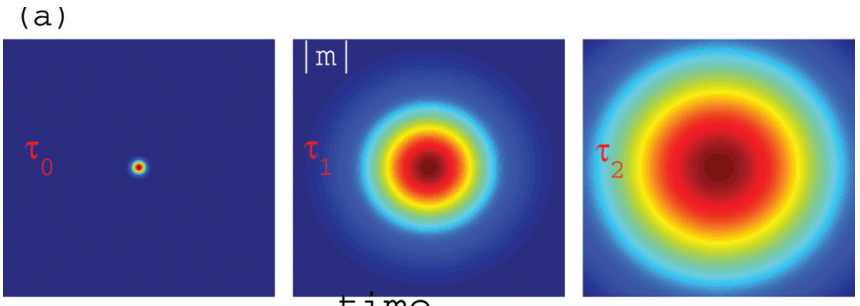

(b)
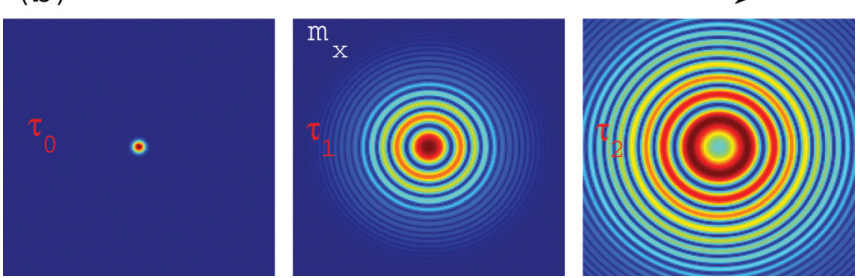

FIG. 3. (Color online) Spatial-dependent excitation of the magnetic moments, $|m|$, in (a) and $m_{x}$ in (b), in the ferromagnetic thin film. A single excitation of a pulsed current (at time $\tau_{0}$ ) spreads and damps out as it moves away from the contact (times $\tau_{2}>\tau_{1}>\tau_{0}$ are plotted).
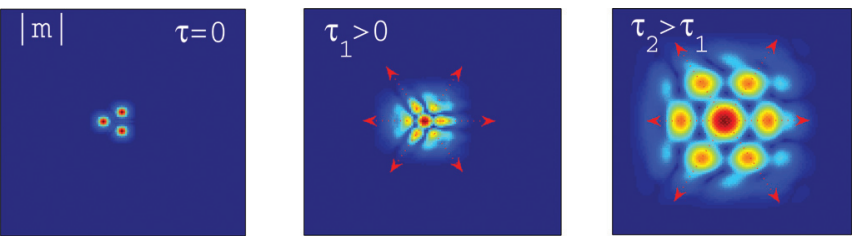

FIG. 4. (Color online) Spatial-dependent excitation of the magnetic moments, $|m|$, in the ferromagnetic thin film. The three point-contacts in a triangular arrangement are excited with a current pulse. Simulations are performed in a 500 by $500 \mathrm{~nm}$ film with point-contacts of $20 \mathrm{~nm}$ diameter and separation between contacts of $60 \mathrm{~nm}$. 

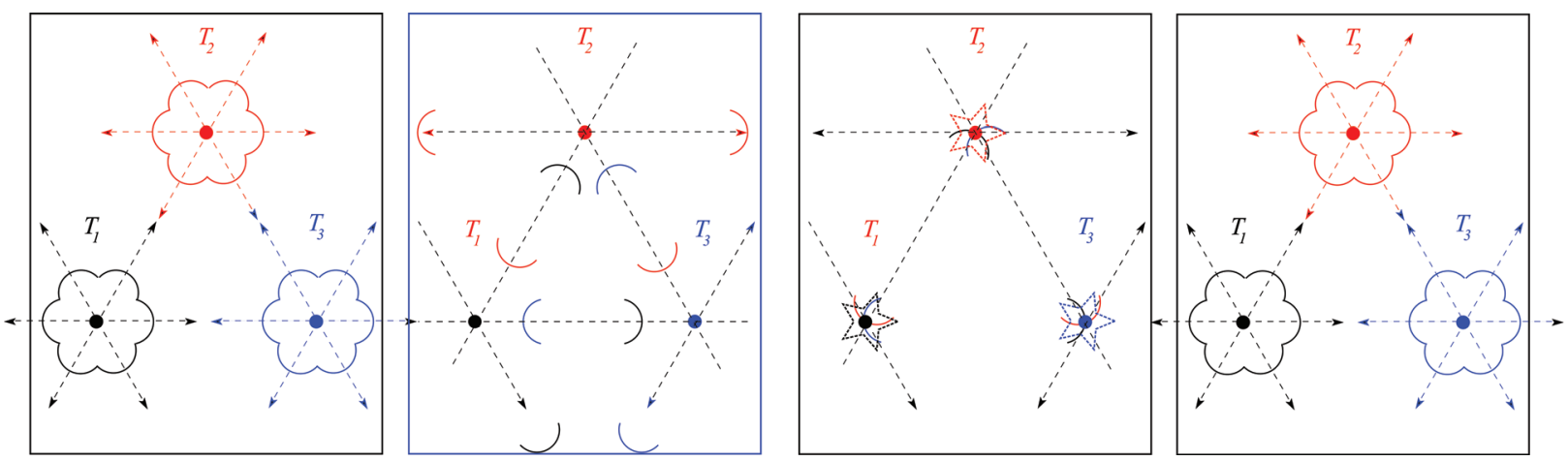

FIG. 5. (Color online) A configuration requiring converging and intersecting wave packets; transponders spike at the same time, the wave-front packets propagate from one to the others and intersect at each transponder reaching the detector threshold and creating new waves. The pattern show repeats periodically.

of inputs, from other transponders, is exceeded. In this case, the transponder may generate a new current induced spinwave excitation, perhaps after some time delay.

STNOs are used to create microwave-frequency excitations in thin films and the same (or other) contacts can also serve as detectors. STNOs may be used to read the state of the system (e.g., the amount of spin-wave activity, arriving spinwave packets, etc.). The respective alignment of the fixed and free layers of an STNO determines its electrical resistance. Thus, small currents (that do not excite magnetization dynamics) through the point-contacts would serve to read the state of the free layer. In order to enhance the readout signal, a magnetic tunnel junction (MTJ) can be used as a detector (see Ref. 12).

In Ref. 12, we described a possible scheme for transponders that would detect spin-wave activity in the propagating medium and respond by initiating new spin-waves. They are basically integrate-and-fire circuits, ${ }^{18,19}$ where a storage device (e.g., a capacitor or an inductor) accumulates charge that is discharged rapidly when a certain threshold level is achieved.

To add complexity to the system and hence create information-processing capacity, configurations that can maintain stable reverberating activity are needed. Detectors may be set to a threshold only reachable through more-than-one arriving energy packets. In Fig. 5 reverberating memory using three transponders is shown. Transponders spike at the same time the wave-front packets propagate from one to the others and intersect at each transponder reaching the detector threshold and creating new waves. Reverberation loops are closed and the activity is periodic with a period depending on the distance between transponders.

The state of the unit is time-dependent. We can encode information in the phase (i.e., timing) of excitation relative to some external timing signal. Any real number could be encoded into the phase of reverberating activity (bounded by the noise in the physical implementation of the system).

Another application would be programming transponder arrangements having graded reverberating frequencies to create a look-up table, similar to the ones in the brain. Consider the analogy of a piano keyboard; an array of several devices as in Figs. 3(a) or 3(b) having a gradient of natural frequencies (i.e., different separations between nodes in reverberators result in different resonant frequencies) are simultaneously exposed to a double-spike signal with period $\tau$. The device with resonant interspike interval $\tau$ will respond coherently, while the others will not. Namely, the double spike will not match with the resonant frequency of others and will create complex reverberating activity from which the coherent one can be distinguished.

Summarizing, we have proposed a design for magnetic nanoscale elements in ferromagnetic thin films that can create controllable spin-wave patterns. The propagating spinwave packets may be used to accomplish some brainlike behaviors through PWC, but on much shorter time scales and on smaller spatial scales. Micromagnetic simulations offer a useful way to design optimal STNO arrangements for specific interference, both steady and propagating patterns.

Supported in part by FENA/FCRP Grant No. 6160-GFD211 and by ARO-MURI, Grant No. W911NF-08-1-0317. F.M. acknowledges support from a Beatriu de Pinós grant from the Catalan Government.

\footnotetext{
${ }^{1}$ M. Tsoi et al., Nature 406, 46 (2000).

${ }^{2}$ S. I. Kiselev et al., Nature 425, 380 (2003).

${ }^{3}$ W. H. Rippard et al., Phys. Rev. Lett. 92, 27201 (2004).

${ }^{4}$ J. C. J. Slonczewski, Magn. Magn. Mater. 159, L1 (1996).

${ }^{5}$ L. Berger, Phys. Rev. B 54, 9353 (1996).

${ }^{6}$ J. C. J. Slonczewski, Magn. Magn. Mater. 195, L261 (1999).

${ }^{7}$ S. Kaka et al., Nature 437, 389 (2005).

${ }^{8}$ F. B. Mancoff, N. D. Rizzo, B. N. Engel, S. Tehrani, Nature 437, 393 (2005).

${ }^{9}$ J. Grollier et al., Phys. Rev. B 67, 174402 (2003).

${ }^{10}$ M. R. Pufall et al., Phys. Rev. Lett. 97, 87206 (2006).

${ }^{11}$ E. M. Izhikevich et al., Int. J. Bifurcation Chaos Appl. Sci. Eng. 19, 1733 (2009).

${ }^{12}$ F. Macià et al., Nanotechnology 22, 095301 (2011).

${ }^{13}$ A. N. Slavin and V. S. Tiberkevich, Phys. Rev. B 74, 10440 (2006).

${ }^{14}$ S. K. Choi, K. S. Lee, and S. K. Kim, Appl. Phys. Lett. 89, 062501 (2006).

${ }^{15}$ S. Choi et al., Appl. Phys. Lett. 90, 083114 (2007).

${ }^{16}$ S. Bance et al., J. Appl. Phys 103, 07E735 (2008).

${ }^{17}$ K. S. Lee, and S. K. J. Kim, Appl. Phys. 104, 053909 (2008).

${ }^{18}$ E. M. Izhikevich, Dynamical Systems in Neuroscience: The Geometry of Excitability and Bursting (The MIT Press, London, 2007).

${ }^{19}$ F. C. Hoppensteadt and E. M. Izhikevich, Weakly Connected Neural Networks (Springer-Verlag, New York, 1997).
} 